\title{
Implications of Twilight Sky Brightness Measurements on Fajr Prayer and Young Crescent Observation
}

\author{
Dhani Herdiwijaya \\ Astronomy Research Division and Bosscha Observatory, Bandung Institute of Technology, Ganesha 10 \\ Bandung, Indonesia 40132 \\ E-mail: dhani@as.itb.ac.id
}

\begin{abstract}
The morning and evening twilight brightness are important to probe the properties of the upper atmosphere. We conducted a short survey of broad band twilight sky brightness measurements by using a portable photometer with 5 second resolution, in five places with different elevations. The darkest zenith sky brightness reached 22.9 MPASS. We found the complex twilight sky brightness variation and proposed 17 degree solar dip for Fajr prayer. The observable young crescent moon which has less than 24 hours in age has the sky brightness at the range of 8-16 magnitude/arcsec ${ }^{2}$. These correspond to telescope visual limiting magnitude of 2.9 to 9.3 . The excellent quality of telescope is needed for this purpose to cope with very low contrast of young crescent moon.
\end{abstract}

Keywords: twilight, sky brightness, fajr prayer, young crescent moon.

\section{Introduction}

The sky brightness refers to the residual light that is present in the night sky during dark, moonless nights and becomes one of the parameters for observing location. It poses a major source of noise for groundbased astronomical observations, and good astronomical sites $^{1}$. In the visible light regime, the sky brightness is influenced by air-glow, direct and scattered starlight, zodiacal light, and light pollution from man-made artificial light. The light pollution can extend many kilometres beyond the boundaries of the city, as can be seen in the worldwide atlas of night sky brightness ${ }^{2}$. The elevation above sea level of the site has an effect on the absorption and the scattering of starlight and air-glow. It is also a function of time on the time-scale of years (e.g. solar activity cycle, which influences the amount of airglow) as well as hours, as the air-glow decreases during the night ${ }^{3}$. Twilight brightness is also important for probing the upper atmosphere where the interaction of the Sun energy comes for the first or the last time. Moreover, the study of the twilight brightness will lead to the determination of two of five daily Islamic compulsory prayer times which are Fajr and Isha prayers.

The sky brightness measurements performed using a portable photometer, Unihedron Sky Quality Meter (SQM). SQM is a photometer pocket-sized, lightweight, and relatively inexpensive with USB connection. The measurement angle is 20 degree and has a relative error of less than $3 \%$. This is what makes SQM can be used easily by the general public to get a quality night sky quantitatively ${ }^{4}$. Temporal resolution of data retrieval can be performed every second, but we set to 5 second. All of the above features are advantage points compared to the CCD measurements. This photometer has a lens and CM500 HOYA filter, so that SQM spectral range is between $320-720 \mathrm{~nm}$. The output of this unit is sky brightness in magnitudes per square arcsecond (MPASS) where high scores reflect the dark sky.

This paper will discuss twilight brightness measurement from five places with different elevations.

\section{Morning Twilight Brightness}

The magnitudes of sky brightness were measured at zenith direction for five places can be seen in Fig. 1 to 
Fig. 5. The darkest sky with $22.89 \mathrm{mag} / \operatorname{arcsec}^{2}$ was found on May 10, 2013 00:20 LT at Kupang. The second place was at Bosscha Observatory, Lembang with $19.68 \mathrm{mag} / \mathrm{arcsec}^{2}$ on May 17, 2013 00:46 LT. Others cities (Bandung, Cimahi and Yogyakarta) showed heavy light pollution. The passing clouds were easily detected with 5 second data resolution. We obtained two points difference to magnify their variations and smoothed with 50 points moving average line.

Before sunrise and after sunset there are intervals of time or twilight in which sunlight reflected by upper atmosphere. The amount of natural light depends on structure, chemical composition, and electricity or sky emission of the upper atmosphere and local weather condition $^{5}$. In general, we define three kinds of twilight based on geometrically angles of the center of the Sun's disk below the horizon, which are 6, 12, and 18 degress solar dip for civil, nautical, and astronomical twilight, respectively. In Islamic country, the twilight time determines two essential prayer times, namely Fajr and Isha, and starting time of fasting. Unfortunately, there is no world consensus for solar dip in both prayer times. Many Islamic countries use solar dip within interval 15 to 20 degrees. In Indonesia, the largest solar dip of 20 degree is accepted for starting morning twilight or Fajr prayer. In top panel of all figures, the astronomical twilight is shown in dashed line and it is about 8 minutes later than 20 degree solar dip (bolt line). However, it seems there were very small sky brightness variations for both two angles. In Bandung, Cimahi, and Kupang, the astronomical twilight indicates the starting of declining magnitude in sky brightness or starting of brighter sky. It is note that Bandung and Cimahi face heavy city light pollution. On the other hand, in Lembang and Jogyakarta, the nautical twilight refers to the starting of brighter sky. So, it is shown that the elevation is not crucial parameter for twilight sky. The study of aerosol composition and sky emission should be done further. For example, the aerosol from eruption of Merapi mount may affect the sky brightness in Jogyakarta. Eruption of Ratu crater in Tangkuban Perahu mount may also contribute to twilight brightness. More complex morning twilight brightness is seen at Kupang in Fig. 5. There were five gradient of changing sky brightness which may correspond to the upper atmospheric composition, since there is no volcanic activities on the whole island. In all places, sky brightness after civil twilight has the same pattern in which the Sun light has already become a dominant effect. In the future, the attention should be carefully taken in between 18 degree (astronomical twilight) and 12 degree (nautical twilight) solar dip.

As it is clearly seen from the above results, so we propose of using 17 degree solar dip for Fajr prayer in Indonesia, instead of 20 degree. It is about 11 minute differences. Although, some area in Indonesia may refer to 15 degree.

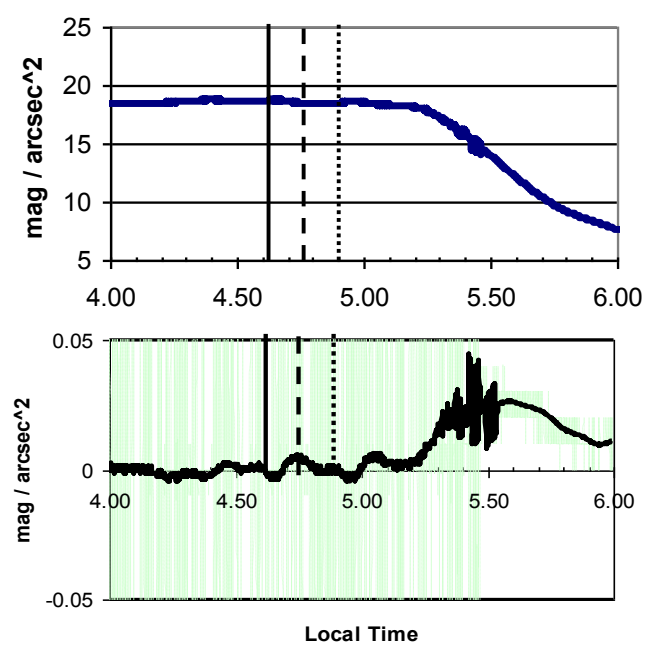

Fig. 1. Zenith morning twilight brightness (top) and its difference with moving average line (bottom) on May 17, 2013 at Lembang ( $107^{0} 37^{\prime} \mathrm{E}$; $6^{0} 49^{\prime} \mathrm{S}$; elev. $\left.\sim 1300 \mathrm{~m}\right)$. The bold, dashed and dot lines refer to $20^{\circ}, 18^{\circ}$, and $16^{\circ}$ solar dip, respectively. 

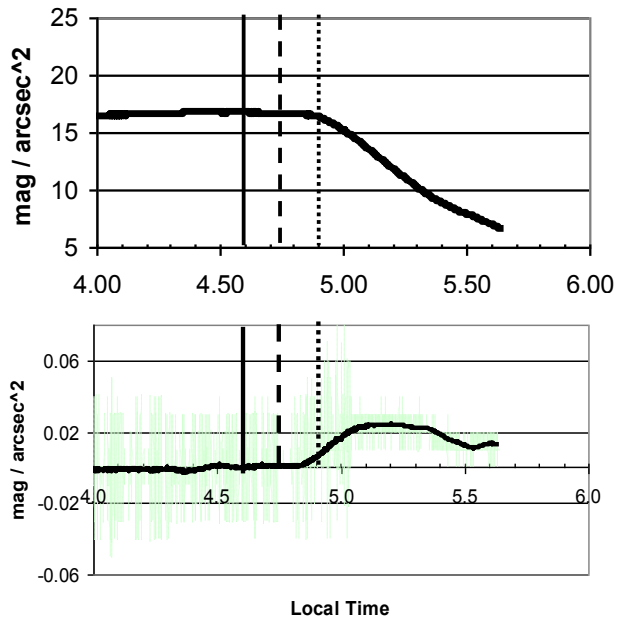

Fig. 2. Zenith morning twilight brightness (top) and its difference with moving average line on Dec. 18, 2013 at Bandung ( $107^{0} 36^{\prime} \mathrm{E}$; $6^{0} 54^{\prime} \mathrm{S}$; elev. $\left.780 \mathrm{~m}\right)$. The bold, dashed and dot lines refer to $20^{\circ}, 18^{\circ}$, and $16^{\circ}$ solar dip, respectively.
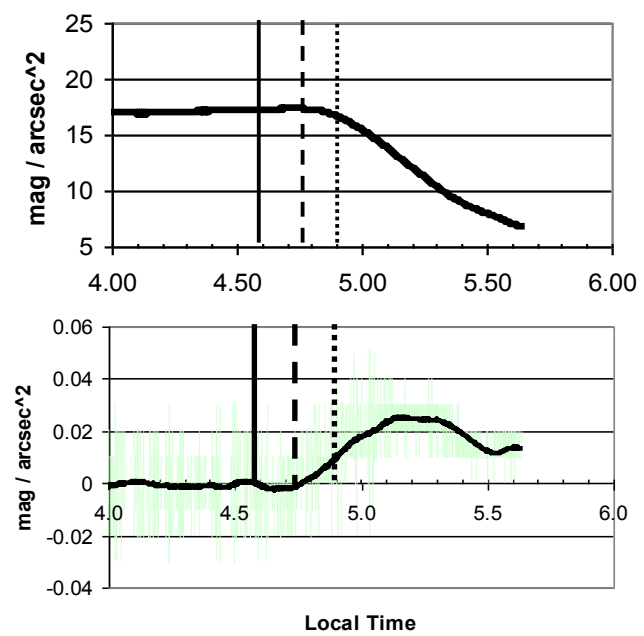

Fig. 3. Zenith morning twilight brightness (top) and its difference with moving average line (bottom) on Dec. 18 , 2013 at Cimahi $\left(107^{0} 32^{\prime} \mathrm{E}\right.$; $6^{0} 53^{\prime} \mathrm{S}$; elev. $\left.700 \mathrm{~m}\right)$. The bold, dashed and dot lines refer to $20^{\circ}, 18^{\circ}$, and $16^{\circ}$ solar dip, respectively.
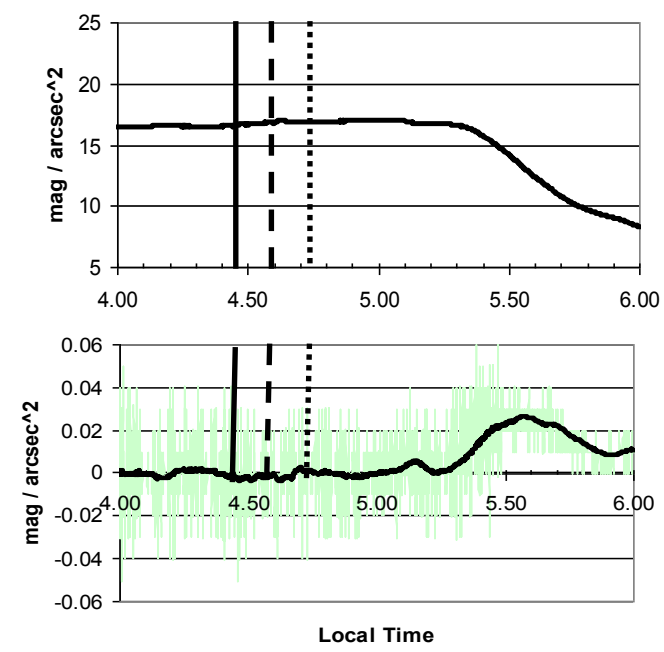

Fig. 4. Zenith morning twilight brightness (top) and its difference with moving average line (bottom) on Jul. 26, 2014 at Jogyakarta $\left(110^{0} 25^{\prime} \mathrm{E} ; 7^{0} 52^{\prime}\right.$ ' S; elev. $\left.100 \mathrm{~m}\right)$. The bold, dashed and dot lines refer to $20^{\circ}, 18^{\circ}$, and $16^{\circ}$ solar dip, respectively.
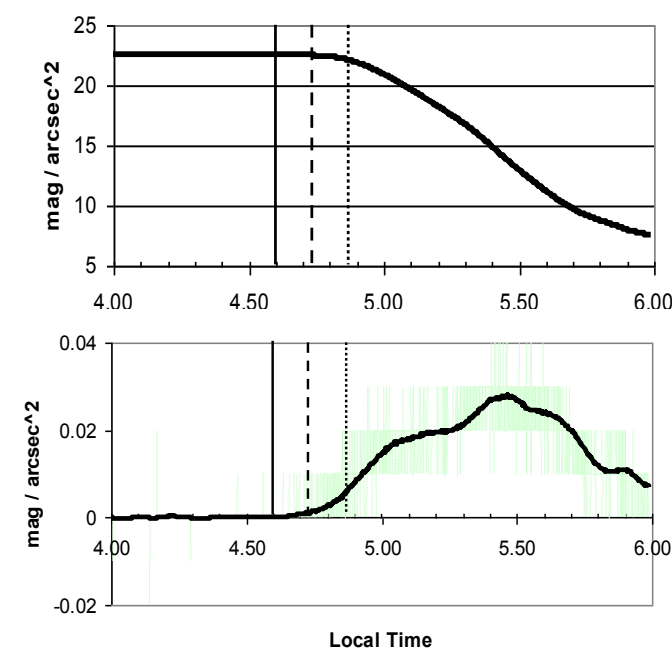

Fig. 5. Zenith morning twilight brightness (top) and its difference with moving average line (bottom) on May 10, 2013 at Amfoang, Kupang (124 0'E; $9^{0} 40^{\prime}$ S; elev. 1300 $\mathrm{m})$. The bold, dashed and dot lines refer to $20^{\circ}, 18^{\circ}$, and $16^{0}$ solar dip, respectively. 


\section{Evening Twilight Brightness}

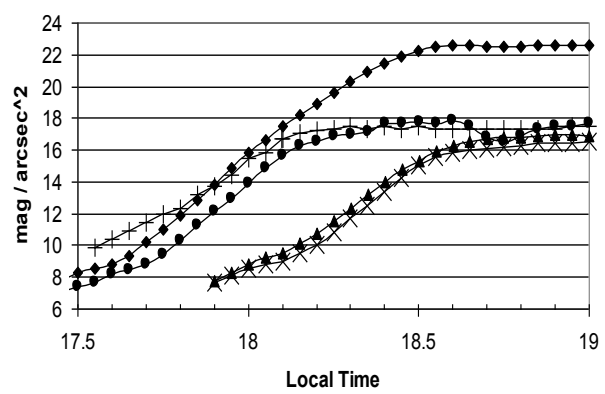

Fig. 6. Zenith evening twilight brightness for five places. Symbol (\$): Kupang (9 May 2013); (+): Lembang (16 May 2013); (•): Jogyakarta (25 Jul. 2014); ( $\mathbf{\Delta})$ : Cimahi (17 Dec. 2013); (×): Bandung (17 Dec. 2013).

From Fig. 6, Cimahi and Bandung cities had the same pattern of bright evening twilight due to light pollution. Kupang showed the darkest twilight brightness. The difference magnitudes were $0.3,1.9$, and $7 \mathrm{mag} . / \mathrm{arcsec}^{2}$ at $06.00 \mathrm{pm}$ with Bosscha Observatory (Lembang), Jogyakarta, and Cimahi or Bandung cities, respectively.

The very young crescent moon with less than 24 hours in age will set before $06.30 \mathrm{pm}$. So, Kupang will give much better contrast of young crescent moon than others. So, this study of evening twilight brightness is very useful to find better site for very young crescent moon observation. Using the above evening twilight brightness, we can compute the limiting magnitude of the telescope ${ }^{6}$, as shown in Fig. 7.

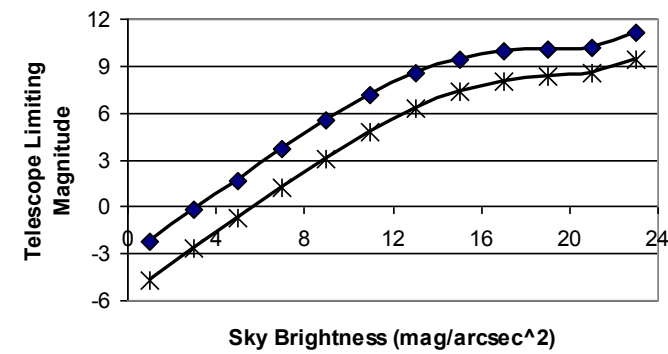

Fig.7. Telescope limiting magnitude for a given sky brightness for zenith angle $=0$ degree (upper) and 80 degree (lower).

After the observational site, the telescope and detector system should be optimized to about 3 magnitudes for low contrast object with high luminosity background with sky brightness of about $8 \mathrm{mag} / \mathrm{arcsec}^{2}$. The near horizon object, e.g. young crescent moon with high zenith angle of 80 degree has lower limiting magnitude than object near zenith, due to higher airmass.

\section{Conclusion}

The morning and evening twilight brightness have been studied for five places with different elevation above sea level. Kupang region showed the darkest sky brightness. The atmospheric composition might give strong influence to the complex variations in twilight brightness. The implication of morning twilight brightness study, we propose 17 degree solar dip for Fajr prayer. On the evening twilight brightness, we suggest to optimize the telescope and detector for observing young crescent moon with known bright background sky brightness.

\section{Acknowledgements}

This work was supported by ITB research grant year 2013.

\section{References}

1. Garstang, R. H., Mar. 1989. Night-sky brightness at observatories and sites. Astron. Soc. of the Pacific 101, 306-329.

2. Cinzano, P., Falchi, F., Elvidge, C. D., Dec. 2001. The first World Atlas of the artificial night sky brightness. Month. Not. Roy. Astr. Soc.328, 689-707.

3. Benn, C. R., Ellison, S. L., Nov. 1998. Brightness of the night sky over La Palma. New Astronomy Reviews 42, 503-507.

4. Cinzano, P. Night Sky Photometry with Sky Quality Meter, ISTIL Internal Report, No. 9, Vol. 1. 4, 2005

5. Rozenberg, G. V. 1966, Twilight: A Study in Atmospheric Optics (New York: Plenum Press), 160.

6. Schaefer, B. E. Telescope Limiting Magnitude. Publ. ASP 102, 212-229. 